QUARTERLY OF APPLIED MATHEMATICS

VOLUME LXV, NUMBER 2

JUNE 2007, PAGES 357-374

S 0033-569X(07)01038-2

Article electronically published on March 5, 2007

\title{
ON THE SHAPE OF THE STABLE PATTERNS FOR ACTIVATOR-INHIBITOR SYSTEMS IN TWO-DIMENSIONAL DOMAINS
}

\author{
$\mathrm{BY}$
}

\author{
YASUHITO MIYAMOTO
}

Research Institute for Mathematical Sciences, Kyoto Univ., Kyoto, 606-8502, Japan

Abstract. We continue to study the shape of the stable steady states of the so-called shadow limit of activator-inhibitor systems in two-dimensional domains

$$
\begin{gathered}
u_{t}=D_{u} \Delta u+f(u, \xi) \text { in } \Omega \times \mathbb{R}_{+} \text {and } \tau \xi_{t}=\frac{1}{|\Omega|} \iint_{\Omega} g(u, \xi) d x d y \text { in } \mathbb{R}_{+}, \\
\partial_{\nu} u=0 \text { on } \partial \Omega \times \mathbb{R}_{+},
\end{gathered}
$$

where $f$ and $g$ satisfy the following: $g_{\xi}<0$, and there is a function $k(\xi) \in C^{0}$ such that $f_{\xi}(u, \xi)=k(\xi) g_{u}(u, \xi)$. This class of reaction-diffusion systems includes the FitzHughNagumo system and a special case of the Gierer-Meinhardt system. In the author's previous paper "An instability criterion for activator-inhibitor systems in a two-dimensional ball" (J. Diff. Eq. 229 (2006), 494-508), we obtain a necessary condition about the profile of $u$ on the boundary of the domain for a steady state $(u, \xi)$ to be stable when the domain is a two-dimensional ball. In this paper, we give a necessary condition about the profile of $u$ in the domain when the domain is a two-dimensional ball, annulus or rectangle. Roughly speaking, we show that if $(u, \xi)$ is stable for some $\tau>0$, then the shape of $u$ is like a boundary one-spike layer even if $D_{u}$ is not small.

1. Introduction. We study the shape of the stable steady states of the so-called shadow system N82 of activator-inhibitor systems in two-dimensional domains

$$
\begin{gathered}
u_{t}=D_{u} \Delta u+f(u, \xi) \text { in } \Omega \times \mathbb{R}_{+} \quad \text { and } \tau \xi_{t}=\frac{1}{|\Omega|} \iint_{\Omega} g(u, \xi) d x d y \text { in } \mathbb{R}_{+}, \\
\partial_{\nu} u=0 \text { on } \partial \Omega \times \mathbb{R}_{+},
\end{gathered}
$$

where the domain $\Omega\left(\subset \mathbb{R}^{2}\right)$ is a ball $B\left(:=B_{r_{1}}\right):=\left\{(x, y) ; x^{2}+y^{2}<r_{1}^{2}\right\}$, an annulus $A:=B_{r_{1}} \backslash\left(B_{r_{0}} \cup \partial B_{r_{0}}\right)\left(0<r_{0}<r_{1}\right)$ or a rectangle $R:=\left\{(x, y) ; 0<x<l_{1}, 0<\right.$

Received May 23, 2006.

2000 Mathematics Subject Classification. Primary 35B35, 35K57; Secondary 35J60, 35P15.

Key words and phrases. Activator-inhibitor system, shadow system, reaction-diffusion system, stability, nodal curve, nodal domain.

E-mail address: miyayan@sepia.ocn.ne.jp 
$\left.y<l_{2}\right\} \quad\left(0<l_{1}, 0<l_{2}\right)$. Here $D_{u}, \tau$ are positive constants, $|\Omega|$ denotes the area of $\Omega$ and $\partial_{\nu}$ denotes the outer normal derivative on $\partial \Omega$. In theoretical biology, the unknowns $u=u(x, t)$ and $\xi=\xi(t)$ stand for the concentrations of biochemicals called the activator and inhibitor respectively. The system $\left(\mathrm{SS}_{\Omega}\right)$ describes various natural phenomena. Two concrete examples of $\left(\mathrm{SS}_{\Omega}\right)$ are given at the end of this section.

Throughout the present paper, we assume that

$$
f(\cdot, \cdot), g(\cdot, \cdot) \text { are of class } C^{2}, g_{\xi}<0 \text { and }
$$

there is a function $k(\xi) \in C^{0}$ such that $f_{\xi}(u, \xi)=k(\xi) g_{u}(u, \xi)$.

This class of reaction-diffusion systems includes the FitzHugh-Nagumo system (Example 1.3 below) and a special case of the Gierer-Meinhardt system (Example 1.4 below). When we consider the case that $\Omega=B$ or $A$, we also assume that

$$
\sup _{\left(\rho_{1}, \rho_{2}\right) \in \mathbb{R}^{2}} f_{u}\left(\rho_{1}, \rho_{2}\right)<D_{u} \kappa_{4}(\Omega),
$$

where $\kappa_{4}(\Omega)$ is the fourth eigenvalue of the Neumann Laplacian

$$
\Delta \phi+\kappa \phi=0 \text { in } \Omega \text { and } \partial_{\nu} \phi=0 \text { on } \partial \Omega .
$$

Here $\kappa_{j}(\Omega)(j \geq 1)$ denotes the eigenvalue of (1.1) counting multiplicities.

A brief statement of our main results is the following;

Theorem 1.1. Let $(u, \xi) \in C^{2} \times \mathbb{R}$ be a non-constant steady state to $\left(\mathrm{SS}_{\Omega}\right)$. Suppose that $(\mathrm{N})$ holds.

(i ) Suppose that $\Omega=B$ and that $\left(\mathrm{F}_{B}\right)$ holds. If $(u, \xi)$ is stable for some $\tau>0$, then there is a line $L$ containing the origin such that $u$ is symmetric with respect to $L$ and the global maximum and minimum of $u$ are attained at $\partial B \cap L$. Moreover, $u$ is strictly monotone in the direction of $L$.

(ii) Suppose that $\Omega=R$. If $(u, \xi)$ is stable for some $\tau>0$, then the global maximum and minimum of $u$ are attained at a corner of $R$ or a side of $R$. Moreover, there is a direction such that $u$ is strictly monotone in the direction.

(iii) Suppose that $\Omega=A$ and that $\left(\mathrm{F}_{A}\right)$ holds. If $(u, \xi)$ is stable for some $\tau>0$, then there is a line $L$ containing the origin such that $u$ is symmetric with respect to $L$ and the global maximum and minimum of $u$ are attained at $\bar{A} \cap L$.

See Theorems $3.3,4.7$ and 4.8 for the precise statements. Theorem 1.1 tells us that only the pattern that the activator concentrates at a point or on a portion of the boundary can be stable when $\Omega=B$ or $R$.

REMARK 1.2. The assumptions $\left(\mathrm{F}_{B}\right)$ and $\left(\mathrm{F}_{A}\right)$ are used to prove the symmetry of the solutions. See Lemma 4.1 and Remark 4.2 in Section 4.

In the case of a single reaction-diffusion equation with the Neumann boundary condition, all the stable steady states are constant when the domain is bounded and convex in $\mathbb{R}^{N}$ CH78, Ma79. In the case of a shadow reaction-diffusion system with the Neumann boundary condition in a one-dimensional interval, all the stable steady states are constant or monotone [N94, NPY01. See [JM94, K05, KY03, L96, Y02a, Y02b, Y02c for other stability and instability results. However, the shape of the stable steady states of a large class of reaction-diffusion systems in high-dimensional domains seems not to be known 
very much. In Mi06, the author has given a necessary condition about the shape of the stable steady states of $\left(\mathrm{SS}_{B_{r_{1}}}\right)$ on the boundary, when the domain is a two-dimensional ball. Specifically, if $(u, \xi)$ is stable for some $\tau>0$, then $u$ is constant or

$$
\mathcal{Z}\left[U_{\theta}(\cdot)\right]=2
$$

Here $U_{\theta}(\theta):=\partial_{\theta}\left(u\left(r_{1} \cos \theta, r_{1} \sin \theta\right)\right)$, and $\mathcal{Z}[\Phi(\cdot)]$ denotes the cardinal number of the zero level set of a function $\Phi(\cdot) \in C^{0}(\mathbb{R} / 2 \pi \mathbb{Z})$. On the other hand, a special case of the Gierer-Meinhardt system in a two-dimensional ball has a stable steady state called $a$ boundary one-spike layer which is a function whose global maximum is attained at exactly one point on the boundary and which almost vanishes outside a neighborhood of the maximum point (see [NTY01, for a two-dimensional ball and see [LNT88, INT91, INT93, Mi05 for general domains). Moreover, this stable boundary one-spike layer satisfies (1.2) [LT01, L01. From the result of [Mi06] we cannot obtain the information of the shape of $u$ in the whole domain. However, the necessary condition (1.2) and the existence of the stable boundary one-spike layer of the Gierer-Meinhardt system seem to suggest that the shape of the stable steady states of $\left(\mathrm{SS}_{B_{r_{1}}}\right)$ is like a boundary one-spike layer. Theorem 1.1 tells us that this expectation is correct provided that $\Omega=B$ or $R$.

It is expected that a similar result holds when the domain is bounded and convex. Specifically, in the case that $\Omega$ is a two-dimensional bounded convex domain, every stable steady state of $\left(\mathrm{SS}_{\Omega}\right)$ attains the global maximum and minimum on the boundary of the domain. However, this seems not to be proved. We discuss a technical point of this conjecture in Section 5.

In GM88, the shape of the global and local minimizers of the functional (5.1) subject to the constraint (5.2) is considered. They obtain results similar to ours when the domain is a two-dimensional ball or rectangle. We discuss this similarity in Section 5 .

Let us explain the system $\left(\mathrm{SS}_{\Omega}\right)$ briefly. This system is a mathematical model describing the interaction of the two biochemicals which are called the activator and inhibitor. The activator activates the production of the inhibitor $\left(g_{u}>0\right)$, and the inhibitor inhibits the production of the activator $\left(f_{\xi}<0\right)$. The inhibitor decays if there is no reaction $\left(g_{\xi}<0\right)$. Hence we call $\left(\mathrm{SS}_{\Omega}\right)$ the shadow limit of the activator-inhibitor system if $f$ and $g$ satisfy

$$
f_{\xi}<0, \quad g_{u}>0 \text { and } g_{\xi}<0 .
$$

The shadow limit describes the situation where the inhibitor diffuses quickly and always becomes spatially homogeneous. The constant $\tau$ means the ratio of the reaction speeds of the activator and inhibitor. If $\tau$ is small, then the inhibitor reacts quickly. In this case the effect that the inhibitor inhibits the activator is strong, and the steady states tend to be stabilized. Thus stable inhomogeneous steady states can exist. On the contrary, if $\tau$ is large, then this effect is weak and the inhibitor $\xi$ changes slowly. In this case $\left(\mathrm{SS}_{\Omega}\right)$ behaves like a single reaction-diffusion equation, and we can easily expect and show that if the domain is convex, all the stable steady states of $\left(\mathrm{SS}_{\Omega}\right)$ are constants. We give two examples satisfying (1.3) and $(\mathrm{N})$. 
ExAmPle 1.3. The shadow system of the FitzHugh-Nagumo model [F61, NAY62] is the following:

$$
u_{t}=D_{u} \Delta u+f_{0}(u)-\alpha \xi \quad \text { and } \quad \tau \xi_{t}=\frac{1}{|\Omega|} \iint_{\Omega}(\beta u-\gamma \xi) d x d y,
$$

where $\alpha, \beta$ and $\gamma$ are positive constants and $f_{0}(u)$ is the so-called cubic function. A typical example of $f_{0}$ is $u(1-u)(u-\delta)(0<\delta<1)$. (1.3) and $(\mathrm{N})$ always hold. $\left(\mathrm{F}_{\Omega}\right)$ holds if $D_{u}$ is large.

EXAMPLE 1.4. The shadow system of the Gierer-Meinhardt model GM72 is the following:

$$
u_{t}=D_{u} \Delta u-u+\frac{u^{p}}{\xi^{q}} \quad \text { and } \quad \tau \xi_{t}=\frac{1}{|\Omega|} \iint_{\Omega}\left(-\xi+\frac{u^{r}}{\xi^{s}}\right) d x d y
$$

where $(p, q, r, s)$ satisfy $p>1, q>0, r>0, s \geq 0$ and $0<(p-1) / q<r /(s+1)$. The assumption on $(p, q, r, s)$ comes from a biological reason. (1.3) always holds. If $r=p+1$, then $(\mathrm{N})$ holds. This system is a model describing head formation of a hydra which is a small creature. Specifically, GM72 shows experimentally that the head appears at the point where the activator $u$ attains the local maximum. It is known that this system has steady states having various shapes (see [NT91, NT93, GW00, MM02 for example). Theorem 1.1 says that if a steady state is stable, then the global maximum of $u$ attains the boundary when $\Omega=B$ or $R$. This result can be interpreted as follows: the head appears at a point on the edge of the body.

We should mention the strategy of the proof of our main results. We do not use the rearrangement technique or the singular perturbation. However, we use the Courant nodal theorem and the zero level sets of $u_{x}, u_{y}$ and $\partial_{\theta}^{\left(x_{0}, y_{0}\right)} u\left(:=-\left(y-y_{0}\right) u_{x}+\left(x-x_{0}\right) u_{y}\right)$, where $(u, \xi)$ is a steady state of $\left(\mathrm{SS}_{\Omega}\right)$. These functions do not necessarily satisfy the Neumann boundary condition. However, we can see the relation between the profile of $u$ and the positivity of the second eigenvalue of the corresponding eigenvalue problem, using a variational technique and these functions. This relation is a key to our analysis.

This paper consists of five sections. In Section 2, we prepare notation and recall known results. In Section 3, we prove the case that $\Omega=R$ (Theorem 3.3). In Section 4, we prove the case that $\Omega=B$ (Theorem 4.7) and that $\Omega=A$ (Theorem 4.8). In Section 5, we discuss a technical difficulty which is Conjecture 5.1 when our result is extended to convex domains. We also discuss the relation between our results and results of GM88.

2. Preliminaries. First, we obtain a sufficient condition for a steady state of ( $\mathrm{SS}_{\Omega}$ ) to be unstable, where the term unstable means that the corresponding eigenvalue problem has an eigenvalue with positive real part. Specifically, we show that a steady state of $\left(\mathrm{SS}_{\Omega}\right)$ is unstable for all $\tau>0$ provided that the second eigenvalue of the eigenvalue problem $\left(\mathrm{LP}_{\Omega}\right)$ below is positive. This result reduces the instability problem of a steady state of the shadow system $\left(\mathrm{SS}_{\Omega}\right)$ to the eigenvalue problem of a single equation $\left(\mathrm{LP}_{\Omega}\right)$.

Second, we recall known results about the zero level set, which is called a nodal line or nodal curve, of the eigenfunction of the eigenvalue problem $\left(\mathrm{LP}_{\Omega}\right)$. The nodal line tells us the relation between the shape of a steady state of non-linear elliptic equations and the Morse index of the steady state which is the number of the positive eigenvalues of the 
corresponding eigenvalue problem. We also recall a strong unique continuation property on the boundary of the domain of linear elliptic equations on two-dimensional domains.

Third, in order to state results about single elliptic equations, we prepare some more notation.

Let $S$ be a subset of $\mathbb{R}^{2}$. From now on, we denote $\operatorname{int}(S), \bar{S}$ by

$$
\operatorname{int}(S):=S \backslash \partial S, \quad \bar{S}:=S \cup \partial S .
$$

2.1. The instability of the steady state of $\left(\mathrm{SS}_{\Omega}\right)$. Let $(u, \xi)$ be a steady state of $\left(\mathrm{SS}_{\Omega}\right)$. In order to establish the instability of the steady state, we consider the eigenvalue problem

$$
\begin{aligned}
& L_{0} v+f_{\xi}(u, \xi) \eta=\lambda v \quad \text { and } \quad\left\langle v, g_{u}(u, \xi)\right\rangle+\left\langle 1, g_{\xi}(u, \xi)\right\rangle \eta=\lambda \tau \eta|\Omega|, \quad\left(\mathrm{EP}_{\Omega}\right) \\
& \partial_{\nu} v=0 \text { on } \partial \Omega \text {, }
\end{aligned}
$$

where $L_{0}:=D_{u} \Delta+f_{u}(u, \xi)$, and $(v, \eta) \in C^{2} \times \mathbb{R}$. We want to prove the existence of the eigenvalue of $\left(\mathrm{EP}_{\Omega}\right)$ with a positive real part. It is important to show that the second eigenvalue of the eigenvalue problem

$$
L_{0} \psi=\chi \psi \text { in } \Omega \text { and } \partial_{\nu} \psi=0 \text { on } \partial \Omega
$$

is positive. From now on, let $\chi_{n}(\Omega)(n \geq 1)$ denote the $n$-th eigenvalue counting multiplicities. We also use $\chi_{n}$ if there is no confusion. Let $\psi_{n}$ denote a corresponding eigenfunction satisfying $\left\|\psi_{n}\right\|_{L^{2}(\Omega)}=1$, and let $\operatorname{spec}\left(L_{0}\right):=\left\{\chi_{n}(\Omega)\right\}_{n=1}^{\infty}$.

Proposition 2.1 (Lemma 3.1 (i) of Mi06]). Let $(u, \xi)$ be a steady state of $\left(\mathrm{SS}_{\Omega}\right)$. Suppose that $(\mathrm{N})$ holds. If $\chi_{2}(\Omega)>0$, then, for all $\tau>0,(u, \xi)$ is unstable. Here $\chi_{2}(\Omega)$ is the second eigenvalue of $\left(\mathrm{LP}_{\Omega}\right)$.

Proposition 2.1 was obtained by Mi06, Y02c]. However, for the completeness of the paper we prove Proposition 2.1.

Proof. We consider $\left(\mathrm{EP}_{\Omega}\right)$. From the second equation of $\left(\overline{\mathrm{EP}_{\Omega}}\right)$, we have

$$
\eta=\frac{\left\langle v, g_{u}(u, \xi)\right\rangle}{\lambda \tau|\Omega|-\left\langle 1, g_{\xi}(u, \xi)\right\rangle} .
$$

We consider the case that $\lambda>0$. Thus $\lambda \tau>0$. Owing to the assumption $(\mathrm{N}), \lambda \tau|\Omega|-$ $\left\langle 1, g_{\xi}(u, \xi)\right\rangle>0$. Thus the denominator of (2.1) does not vanish. Substituting (2.1) into the first equation of $\left(\mathrm{EP}_{\Omega}\right)$, we obtain the eigenvalue problem of $v$,

$$
\left(L_{0}+A_{\lambda, \tau}\right) v=\lambda v
$$

where $A_{\lambda, \tau}$ is a rank-one operator (i.e., $\operatorname{dim} \operatorname{Ran} A_{\lambda, \tau}=1$ ) and

$$
A_{\lambda, \tau} v=\frac{\left\langle v, g_{u}(u, \xi)\right\rangle}{\lambda \tau|\Omega|-\left\langle 1, g_{\xi}(u, \xi)\right\rangle} f_{\xi}(u, \xi) \text {. }
$$

Note that $\lambda$ appears in $A_{\lambda, \tau}$. Thus (2.2) is not a standard eigenvalue problem. We see by the Sherman-Morrison formula that

$$
\left(L_{0}+A_{\lambda, \tau}-\lambda\right)^{-1}=\left(1-\frac{\left(L_{0}-\lambda\right)^{-1} A_{\lambda, \tau}}{h(\lambda)}\right)\left(L_{0}-\lambda\right)^{-1},
$$

where

$$
h(\lambda)=1+\frac{\left\langle\left(L_{0}-\lambda\right)^{-1}\left[f_{\xi}(u, \xi)\right], g_{u}(u, \xi)\right\rangle}{\lambda \tau|\Omega|-\left\langle 1, g_{\xi}(u, \xi)\right\rangle} .
$$


Therefore under the condition that $\lambda \notin \operatorname{spec}\left(L_{0}\right), L_{0}+A_{\lambda, \tau}-\lambda$ is invertible if and only if

$$
h(\lambda) \neq 0 .
$$

On the other hand, from the eigenfunction expansion we see

$$
\left(L_{0}-\lambda\right)^{-1}[\phi]=-\sum_{n \geq 1} \frac{\left\langle\phi, \psi_{n}\right\rangle}{\lambda-\chi_{n}} \psi_{n} .
$$

Substituting (2.3) into $h(\lambda)=0$, we obtain the following equation:

$$
\lambda \tau|\Omega|-\left\langle 1, g_{\xi}(u, \xi)\right\rangle=h_{0}(\lambda),
$$

where

$$
h_{0}(\lambda)=\sum_{n \geq 1} \frac{a_{n}}{\lambda-\chi_{n}} \quad \text { and } \quad a_{n}=\left\langle f_{\xi}(u, \xi), \psi_{n}\right\rangle\left\langle\psi_{n}, g_{u}(u, \xi)\right\rangle .
$$

We divide this case into three more cases.

Case $k(\xi)=0$ : In this case, $a_{1}=0$. Thus $\left(L+A_{\chi_{1}, \tau}-\chi_{1}\right)\left[\psi_{1}\right]=0$, which indicates that $\chi_{1}\left(>\chi_{2}>0\right)$ is an eigenvalue of $\left(\mathrm{EP}_{\Omega}\right)$. Thus $(u, \xi)$ is unstable.

Case $k(\xi)<0$ : In this case, $a_{1}<0$. If $a_{2} \neq 0$, then $a_{2}<0$. Thus $\lim _{\lambda \uparrow \chi_{1}} h_{0}(\lambda)=$ $+\infty, \lim _{\lambda \downarrow \chi_{2}} h_{0}(\lambda)=-\infty$ and $h(\lambda) \in C^{0}\left(\left(\chi_{2}, \chi_{1}\right)\right)$. Therefore, for any $\tau>0$, there is $\tilde{\lambda} \in\left(\chi_{2}, \chi_{1}\right)$ such that $\tilde{\lambda} \tau|\Omega|-\left\langle 1, g_{\xi}\right\rangle=h_{0}(\tilde{\lambda})$, which indicates that $(u, \xi)$ is unstable.

If $a_{2}=0$, then $\left(L+A_{\chi_{2}, \tau}-\chi_{2}\right)\left[\psi_{2}\right]=0$. Thus $\chi_{2}(>0)$ is an eigenvalue of $\left(\mathrm{EP}_{\Omega}\right)$. Thus $(u, \xi)$ is unstable.

Case $k(\xi)>0$ : Since $a_{1}>0, \lim _{\lambda_{\downarrow} \chi_{1}} h_{0}(\lambda)=+\infty, \lim _{\lambda \uparrow+\infty} h_{0}(\lambda)=0$ and $h(\lambda) \in$ $C^{0}\left(\left(\chi_{1},+\infty\right)\right)$. Hence, for any $\tau>0$, there is $\tilde{\lambda}\left(>\chi_{2}>0\right)$ such that $\tilde{\lambda} \tau|\Omega|-\left\langle 1, g_{\xi}\right\rangle=$ $h_{0}(\tilde{\lambda})$. Thus $(u, \xi)$ is unstable.

2.2. The zero level set of the eigenfunction. Hereafter, we identify a complex number $\zeta$ with a point in the two-dimensional domain $\Omega$. Let $z(\zeta)(\zeta \in \mathbb{C})$ be a real-valued function satisfying the following:

$$
\text { there is a constant } C>0 \text { such that }|\Delta z| \leq C(|\nabla z|+|z|) \text {. }
$$

Using the Carleman-Hartman-Wintner theory, we can see the local behavior of the function $z(\zeta)$ satisfying (2.4).

Proposition 2.2 ([HW53, C33 $)$. Let $z(\zeta)=o\left(|\zeta|^{n+1}\right)$ as $|\zeta| \rightarrow 0$ for some $n \in \mathbb{N}$. If $z(\zeta)$ satisfies (2.4), then either (i) or (ii) holds:

(i) $z(\zeta) \equiv 0$ in $\Omega$,

(ii) there exists an integer $m(\geq n)$ such that

$$
(A:=) \frac{2}{m+1} \lim _{\substack{|\zeta| \rightarrow 0 \\ \zeta \neq 0}} \frac{z_{\zeta}(\zeta)}{\zeta^{m}} \neq 0,
$$

and $z(\zeta)$ has an asymptotic expansion of the form

$$
z(\zeta)=\operatorname{Re}\left(A \zeta^{m+1}\right)+o\left(|\zeta|^{m+1}\right) \text { as } \zeta \rightarrow 0,
$$

where $\operatorname{Re}\left(A \zeta^{m+1}\right)$ is the real part of the complex function $A \zeta^{m+1}$. 
REMARK 2.3. The real-valued function $\operatorname{Re}\left(A \zeta^{m}\right)$ has a particularly simple representation in polar coordinates $(\rho, \omega)$ :

$$
\operatorname{Re}\left(A \zeta^{m}\right)=\alpha \rho^{m} \cos (m \omega)+\beta \rho^{m} \sin (m \omega)
$$

where $\alpha, \beta \in \mathbb{R}$. Obviously the zero level set of $\operatorname{Re}\left(A \zeta^{m}\right)$ consists of $m$ straight lines which meet at an equal angle. See [HMN99] for details.

Let $\phi$ be an eigenfunction of $\left(\overline{\mathrm{LP}_{\Omega}}\right)$. Then $D_{u} \Delta \phi=\left(\mu-f_{u}(u, \xi)\right) \phi$, and $\phi$ satisfies (2.4). Proposition 2.2 can be applied to $\phi$, and we obtain the following:

Proposition 2.4. The zero level set of $\phi$, which is a solution of $\left(\overline{\mathrm{LP}_{\Omega}}\right)$, consists of either the whole domain or $C^{1}$-curves and intersections among those curves.

Because of Proposition 2.4 we call the zero level set of $\phi,\{(x, y) ; \phi(x, y)=0\}, a$ nodal curve. We call each connected component of $\{(x, y) ; \phi(x, y) \neq 0\}$ a nodal domain.

In Sections 3 and 4, we use the following corollary of Proposition 2.2 .

Corollary 2.5. Let $V(x, y) \in C^{0}(\Omega)$, and let $\phi(x, y)$ be a function satisfying $D_{u} \Delta \phi+$ $V \phi=0$ in $\Omega$. If there exists $\left(x_{0}, y_{0}\right) \in \operatorname{int}(\Omega)$ such that $\phi\left(x_{0}, y_{0}\right)=\phi_{x}\left(x_{0}, y_{0}\right)=$ $\phi_{y}\left(x_{0}, y_{0}\right)=0$ (or $\left.\phi\left(x_{0}, y_{0}\right)=0\right)$, then either (i) or (ii) holds:

(i) $\phi \equiv 0$ in $\Omega$,

(ii) the zero level $\{(x, y) ; \phi(x, y)=0\}$ has at least four (or two respectively) branches at $\left(x_{0}, y_{0}\right)$. Moreover, there are at least two connected components of $\{(x, y) ; \phi(x, y) \neq$ $0\}$, which are $\{(x, y) ; \phi(x, y)>0\}$ and $\{(x, y) ; \phi(x, y)<0\}$, near the point $\left(x_{0}, y_{0}\right)$.

The following proposition is a strong continuation property of linear elliptic equations on the boundary of the domain.

Proposition 2.6 (Lemma 4.4 of [Mi06]). Let $\Omega\left(\subset \mathbb{R}^{2}\right)$ be a bounded domain, and let $\Gamma(\subset \partial \Omega)$ be a portion of the boundary of class $C^{2}$. Let $V \in C^{0}(\Omega)$. If there is a neighborhood of $\Gamma$ in $\Omega, U$, and a function $\phi \in C^{2}(U) \cap C^{1}(\bar{U})$ such that

$$
D_{u} \Delta \phi+V \phi=0 \text { in } U \text { and } \phi=\partial_{\nu} \phi=\partial_{\tau} \phi=0 \text { on } \Gamma,
$$

then $\phi \equiv 0$ in $U$, where $\partial_{\tau} \phi$ is the tangential derivative along $\Gamma$.

Proposition 2.7 (Lemma 4.3 of [Mi06]). Let $\Omega\left(\subset \mathbb{R}^{2}\right)$ be a bounded domain with piecewise $C^{2}$ boundary, and let $V \in C^{0}(\Omega)$. Let $\phi$ be a non-trivial solution to

$$
D_{u} \Delta \phi+V \phi=0 \text { in } \Omega \text { and } \partial_{\nu} \phi=0 \text { on } \partial \Omega .
$$

Suppose that there is $\left(x_{0}, y_{0}\right) \in \partial \Omega$ such that $\phi\left(x_{0}, y_{0}\right)=0$ and that $\partial \Omega$ is of class $C^{2}$ near $\left(x_{0}, y_{0}\right)$. Then there is a nodal curve of $\phi$ connecting to $\left(x_{0}, y_{0}\right)$.

2.3. Notation. By $L^{2}(\Omega), H^{1}(\Omega)$ we denote the usual Lebesgue space and Sobolev space of order one on $\Omega$ respectively. By $\langle\cdot, \cdot\rangle,\|\cdot\|_{L^{2}(\Omega)}$ we denote the inner product and norm of $L^{2}(\Omega)$ respectively, i.e.,

$$
\langle F, G\rangle:=\iint_{\Omega} F(x, y) G(x, y) d x d y \quad \text { and } \quad\|F\|_{L^{2}(\Omega)}:=\sqrt{\langle F, F\rangle} .
$$




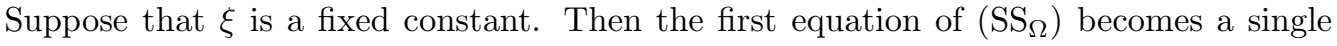
elliptic equation of $u$. In Sections 3 and 4, we consider this single equation, and use a different notation as follows:

$$
D_{u} \Delta w+N(w)=0 \text { in } \Omega \text { and } \partial_{\nu} w=0 \text { on } \partial \Omega,
$$

where $N(\cdot)$ is of class $C^{2}$. Let $w$ be a solution of $\left(\mathrm{SE}_{\Omega}\right)$. Then the corresponding eigenvalue problem becomes as follows:

$$
D_{u} \Delta \psi+N^{\prime}(w) \psi=\mu \psi \text { in } \Omega \text { and } \partial_{\nu} \psi=0 \text { on } \partial \Omega \text {. }
$$

Hereafter, by $\mu_{n}(\Omega)(n \geq 1)$ we denote the $n$-th eigenvalue of $\left(\mathrm{EPSE}_{\Omega}\right)$ counting multiplicities. We call the number $\sharp\left\{\mu_{n}(\Omega)>0\right\}$ the Morse index of $w$. When we consider the problem $\left(\mathrm{SE}_{\Omega}\right)$, the assumption $\left(\mathrm{F}_{\Omega}\right)$ is read as follows:

$$
\sup _{\rho \in \mathbb{R}} N^{\prime}(\rho)<D_{u} \kappa_{4}(\Omega),
$$

where $\kappa_{4}(\Omega)$ is the fourth eigenvalue of (1.1).

3. Rectangle. In this section we consider the case that the domain is a rectangle, i.e., $\Omega=R$. Specifically, the purpose of this section is to prove Theorem 3.3 below.

First, we give a sufficient condition for the second eigenvalue of $\left(\mathrm{EPSE}_{R}\right)$ to be positive. In the proof we use a variational characterization of the second eigenvalue of $\left(\mathrm{EPSE}_{\Omega}\right)$

$$
\mu_{2}(\Omega):=\sup _{z \in\left(\operatorname{span}\left\langle\psi_{1}\right\rangle^{\perp} \cap H^{1}(\Omega)\right)} \mathcal{H}_{\Omega}[z] /\|z\|_{L^{2}(\Omega)}^{2},
$$

where $\psi_{1}$ is an eigenfunction corresponding to the first eigenvalue of (EPSE $\Omega$,

$$
\operatorname{span}\left\langle\psi_{1}\right\rangle^{\perp}:=\left\{z \in L^{2}(\Omega) ;\left\langle z, \psi_{1}\right\rangle=0\right\}
$$

and

$$
\begin{aligned}
\mathcal{H}_{\Omega}[\phi] & :=\iint_{\Omega}\left(-D_{u}|\nabla \phi|^{2}+N^{\prime}(w) \phi^{2}\right) d x d y \\
& =\iint_{\Omega} \phi\left(D_{u} \Delta \phi+N^{\prime}(w) \phi\right) d x d y-D_{u} \int_{\partial \Omega} \phi\left(\partial_{\nu} \phi\right) d \sigma .
\end{aligned}
$$

We define the set $R_{1}, R_{2}$ by

$$
R_{1}:=\left\{(0, y),\left(l_{1}, y\right) ; 0 \leq y \leq l_{2}\right\}, \quad R_{2}:=\left\{(x, 0),\left(x, l_{2}\right) ; 0 \leq x \leq l_{1}\right\} .
$$

Lemma 3.1. Let $w$ be a non-constant solution to $\left(\mathrm{SE}_{R}\right)$, and let $\mu_{2}(R)$ be the second eigenvalue of $\left(\mathrm{EPSE}_{R}\right)$. Then (i) and (ii) hold.

(i) If there exists a point $\left(x_{0}, y_{0}\right) \in \bar{R} \backslash R_{1}$ such that $w_{x}\left(x_{0}, y_{0}\right)=0$, then either $w_{x} \equiv 0$ in $R$ or $\mu_{2}(R)>0$.

(ii) If there exists a point $\left(x_{0}, y_{0}\right) \in \bar{R} \backslash R_{2}$ such that $w_{y}\left(x_{0}, y_{0}\right)=0$, then either $w_{y} \equiv 0$ in $R$ or $\mu_{2}(R)>0$.

Proof. The proofs of (i) and (ii) are the same, and we will prove (i) only. Let $\phi:=w_{x}$. We assume that $\phi \not \equiv 0$ in $R$. From the assumption of the lemma we see that $\phi\left(x_{0}, y_{0}\right)=0$ and that $D_{u} \Delta \phi+N^{\prime}(w) \phi=0$ in $R$. If $\left(x_{0}, y_{0}\right) \in \operatorname{int}(R)$, then we can use Corollary 2.5. 
If $\left(x_{0}, y_{0}\right) \in R_{2}$, then we can use Proposition 2.7. Whichever case occurs, $\phi$ has at least two nodal domains which are $\{\phi>0\}$ and $\{\phi<0\}$. We define $\phi^{+}, \phi^{-}$by

$$
\phi^{+}(x, y):=\left\{\begin{array}{ll}
\phi(x, y) & \text { in }\{(x, y) ; \phi(x, y)>0\}, \\
0 & \text { in }\{(x, y) ; \phi(x, y) \leq 0\},
\end{array} \quad \phi^{-}(x, y):=\phi^{+}(x, y)-\phi(x, y) .\right.
$$

Let $\psi_{1}$ be the first eigenfunction of $\left(\operatorname{EPSE}_{R}\right)$. We define $\phi^{*}$ by $\phi^{*}:=\phi^{+}+\alpha \phi^{-}(\alpha \in \mathbb{R})$, where $\alpha$ is chosen so that $\left\langle\phi^{*}, \psi_{1}\right\rangle=0$. Note that $\phi^{*} \in H^{1}(R)$. Then we have

$$
\mathcal{H}_{R}\left[\phi^{*}\right]=-D_{u} \int_{\partial R} \phi^{*}\left(\partial_{\nu} \phi^{*}\right) d \sigma=0
$$

where we use (3.2) and the fact that $\phi^{*}=0$ on $R_{1}$ and that $\partial_{\nu} \phi^{*}=0$ on $R_{2}$. By a variational characterization of the second eigenvalue (3.1) we have that

$$
\mu_{2}(R) \geq \mathcal{H}_{R}\left[\phi^{*}\right] /\left\|\phi^{*}\right\|_{L^{2}(R)}^{2}=0 .
$$

We will show by contradiction that $\mu_{2}(R)>0$. Suppose the contrary. We assume that $\mu_{2}(R)=0$. Then $\phi^{*}$ should be an eigenfunction corresponding to $\mu_{2}(R)$, and satisfy the Neumann boundary condition. Hence $\phi^{*}=\partial_{x} \phi^{*}=0$ on $L_{1}$. By a strong unique continuation on the boundary (Proposition 2.6) we see that $\phi^{*} \equiv 0$ in a neighborhood of a point on $L_{1}$. Moreover it follows from the usual strong continuation at an interior point that $\phi^{*} \equiv 0$ in $R$, which is a contradiction.

Second, we study the shape of a solution of $\left(\mathrm{SE}_{R}\right)$ when the Morse index of the solution is one.

Lemma 3.2. Let $w$ be a non-constant solution to $\left(\mathrm{SE}_{R}\right)$, and let $\mu_{2}(R)$ be the second eigenvalue of $\left(\mathrm{EPSE}_{R}\right)$. If $\mu_{2}(R) \leq 0$, then either (i) or (ii) holds.

(i) There is a direction which is not parallel to the $x$-axis and $y$-axis such that $w$ is strictly monotone with respect to the direction. Moreover $w$ attains its global maximum (minimum) at exactly one point of the corner of $R$.

(ii) $w$ depends only on $x$ or $y$, and it is strictly monotone in $x$ or $y$ respectively. Therefore $w$ attains its global maximum (minimum) on one side of $R$.

Proof. First, we assume that $w_{x} \equiv 0$ in $R$. Let $\phi:=w_{y}$. Then $\phi$ depends only on $y$. The eigenvalue problem $\left(\operatorname{EPSE}_{R}\right)$ becomes a one-dimensional problem and $\phi$ satisfies $D_{u} \phi_{y y}+N^{\prime}(w) \phi=0$ in $R$. Suppose that $\phi$ changes the sign. Then by the SturmLiouville theory we see that the second eigenvalue is positive. This is a contradiction, because $\mu_{2}(R) \leq 0$. Thus $w_{y}$ does not change the sign, and (ii) occurs.

Second, we assume that $w_{x} \not \equiv 0$ in $R$. Then we see by Lemma 3.1(i) that $w_{x}$ does not change the sign, because $\mu_{2} \leq 0$. Thus $w_{x}>0$ in $\bar{R} \backslash \partial R_{1}$ or $w_{x}<0$ in $\bar{R} \backslash \partial R_{1}$. By the same argument as $w_{x}$ we see that there are three cases: $w_{y} \equiv 0$ in $R, w_{y}>0$ in $\bar{R} \backslash \partial R_{2}$, or $w_{y}<0$ in $\bar{R} \backslash \partial R_{2}$. (ii) occurs if $w_{y} \equiv 0$ in $R$, and (i) occurs otherwise.

Theorem 3.3 (Rectangle). Let $\Omega=R$, and let $(u, \xi)$ be a non-constant steady state to $\left(\mathrm{SS}_{R}\right)$. Suppose that $(\mathrm{N})$ holds. If $(u, \xi)$ is stable for some $\tau>0$, then either (i) or (ii) holds. 
(i) There is a direction which is not parallel to the $x$-axis and $y$-axis such that $u$ is strictly monotone with respect to the direction. Moreover $u$ attains its global maximum (minimum) at exactly one point of the corner of $R$.

(ii) $u$ depends only on $x$ or $y$, and it is strictly monotone in $x$ or $y$ respectively. Therefore $u$ attains its global maximum (minimum) on one side of $R$.

Proof. From the assumption of the theorem we see that $(u, \xi)$ is stable for some $\tau>0$. The contrapositive of Proposition 2.1 says that the second eigenvalue of $\left(\mathrm{LP}_{R}\right), \chi_{2}(R)$, is less than or equal to 0 . The desirable result is obtained from Lemma 3.2

4. Ball and annulus. In this section we consider the case that the domain is a ball or an annulus, i.e., $\Omega=B$ or $A$. Specifically, the purpose of this section is to prove Theorems 4.7 and 4.8 below.

Hereafter, we say that $\left(x_{0}, y_{0}\right)$ is a critical point of $w$ if $w_{x}\left(x_{0}, y_{0}\right)=w_{y}\left(x_{0}, y_{0}\right)=$ 0 , and we say that $\left(x_{0}, y_{0}\right)$ is a degenerate point of $w$ if $w\left(x_{0}, y_{0}\right)=w_{x}\left(x_{0}, y_{0}\right)=$ $w_{y}\left(x_{0}, y_{0}\right)=0$.

First, we will prove the symmetry of the solutions of $\left(\mathrm{SE}_{B}\right)$. The condition $\left(\mathrm{F} 0_{B}\right)$ is used to prove the symmetry.

Lemma 4.1. Let $w$ be a solution to $\left(\mathrm{SE}_{B}\right)$. Suppose that $\left(\mathrm{F}_{B}\right)$ holds. Then there is a line containing the center of $B$ such that $w$ has the reflection symmetry with respect to the line.

Proof. Let $w$ be a solution of $\left(\mathrm{SE}_{B}\right)$. Let $R^{(\theta)}$ denote the counterclockwise rotation by $\theta$, and let $S$ be the reflection with respect to the $y$-axis, i.e., $(x, y) \mapsto(-x, y)$. We define $w^{(\theta)}(x, y), \tilde{w}^{(\theta)}(x, y)$ by

$$
w^{(\theta)}(x, y):=\left(R^{(\theta)} w\right)(x, y), \quad \tilde{w}^{(\theta)}(x, y):=\left(R^{(-\theta)} S w\right)(x, y),
$$

namely $w^{(\theta)}(x, y)=w(x \cos \theta+y \sin \theta,-x \sin \theta+y \cos \theta)$ and

$$
\tilde{w}^{(\theta)}(x, y)=w(-x \cos \theta+y \sin \theta, x \sin \theta+y \cos \theta) .
$$

We define $\phi^{(\theta)}$ by

$$
\phi^{(\theta)}:=w^{(\theta)}-\tilde{w}^{(\theta)} .
$$

Then $\phi^{(\theta)}(-x, y)=-\phi^{(\theta)}(x, y)$, and $\phi^{(\theta)}$ satisfies

$$
D_{u} \Delta \phi^{(\theta)}+V \phi^{(\theta)}=0 \text { in } B \text { and } \partial_{\nu} \phi^{(\theta)}=0 \text { on } \partial B,
$$

where

$$
V(x, y):= \begin{cases}\frac{N\left(w^{(\theta)}(x, y)\right)-N\left(\tilde{w}^{(\theta)}(x, y)\right)}{w^{(\theta)}(x, y)-\tilde{w}^{(\theta)}(x, y)} & \text { in }\left\{(x, y) ; w^{(\theta)}(x, y) \neq \tilde{w}^{(\theta)}(x, y)\right\} \\ N^{\prime}\left(w^{(\theta)}(x, y)\right) & \text { in }\left\{(x, y) ; w^{(\theta)}(x, y)=\tilde{w}^{(\theta)}(x, y)\right\} .\end{cases}
$$

Here 0 is an eigenvalue and $\phi^{(\theta)}$ is an associated eigenfunction of the eigenvalue problem

$$
D_{u} \Delta \phi+V \phi=\gamma \phi \text { in } B \text { and } \partial_{\nu} \phi=0 \text { on } \partial B \text {, }
$$

provided that $\phi^{(\theta)} \not \equiv 0$ in $B$. It is clear that $\phi^{(\theta)}=0$ on $B \cap\{(x, y) ; x=0\}$ for all $\theta$. In particular, $\phi^{(\theta)}(0,0)=\phi_{y}^{(\theta)}(0,0)=0$. Since $\phi_{x}^{(\theta)}(0,0)=2\left(w_{x}(0,0) \cos \theta-w_{y}(0,0) \sin \theta\right)$, there exists $\theta_{0} \in[0,2 \pi)$ such that $\phi_{x}^{\left(\theta_{0}\right)}(0,0)=0$. By Corollary 2.5] we see that either 
$\phi^{\left(\theta_{0}\right)} \equiv 0$ in $B$ or $\phi^{\left(\theta_{0}\right)}$ has at least four branches of $\left\{(x, y) ; \phi^{\left(\theta_{0}\right)}(x, y)=0\right\}$ at $(0,0)$. We will show by contradiction that $\phi^{\left(\theta_{0}\right)} \equiv 0$ in $B$. Suppose the contrary. Since the zero level set of $\phi^{\left(\theta_{0}\right)}$ is symmetric with respect to the $y$-axis, there is at least one branch on each side of $B \backslash\{(x, y) ; x=0\}$ and the branch should divide each side into at least two subdomains. Hence $\phi^{\left(\theta_{0}\right)}$ has at least four nodal domains. By the Courant nodal theorem we see that there is an integer $n_{0}(\geq 4)$ such that $\gamma_{n_{0}}=0$, where $\left\{\gamma_{n}\right\}_{n=1}^{\infty}$ is the eigenvalue of (4.2) counting multiplicities. In particular, the fourth eigenvalue is greater than or equal to 0 . However, the fourth eigenvalue of the eigenvalue problem

$$
D_{u} \Delta \phi+D_{u} \kappa_{4}(B) \phi=\mu \phi \text { in } B \text { and } \partial_{\nu} \phi=0 \text { on } \partial B
$$

is 0 . Because of the assumption $\left(\mathrm{F}_{B}\right), V<D_{u} \kappa_{4}(B)$. It follows from the comparison principle of the eigenvalues of linear elliptic partial differential operators that the fourth eigenvalue of (4.2) should be less than the fourth eigenvalue of (4.3), which is 0 . We obtain a contradiction. From the assumption of contradiction we see that there exists $\theta_{0} \in[0,2 \pi)$ such that $\phi^{\left(\theta_{0}\right)} \equiv 0$ in $B$. We obtain the desirable result.

REMARK 4.2. Let $w$ be a solution to $\left(\mathrm{SE}_{A}\right)$, and let $\phi^{(\theta)}$ be a function defined by (4.1). In the case that $\Omega=A$, we can have a similar argument under the condition $\left(\mathrm{F} 0_{A}\right)$. Specifically, there is $\theta_{0} \in[0,2 \pi)$ such that a degenerate point of $\phi^{\left(\theta_{0}\right)}$ appears on $\operatorname{int}(A) \cap\{(x, y) ; x=0\}$. Thus $\phi^{\left(\theta_{0}\right)}$ has at least four nodal domains, which implies that $w$ is symmetric with respect to a line containing the origin.

Lemma 4.3. Let $w$ be a solution of $\left(\mathrm{SE}_{\Omega}\right)$, and let $\phi$ be a non-constant function such that $D_{u} \Delta \phi+N^{\prime}(w) \phi=0$ in $\Omega$. Suppose that there are two nodal domains of $\phi, \Omega_{1}$ and $\Omega_{2}$, and an interior point $p \in \operatorname{int}(\Omega)$ such that the measures (lengths) of $\partial \Omega_{1} \cap \partial \Omega$ and $\partial \Omega_{2} \cap \partial \Omega$ are zero and that $p \notin \overline{\Omega_{1}} \cup \overline{\Omega_{2}}$. Then $\mu_{2}(\Omega)>0$, where $\mu_{2}(\Omega)$ is the second eigenvalue of $\left(\mathrm{EPSE}_{\Omega}\right)$.

Proof. We define $\phi^{(1)}, \phi^{(2)}, \phi^{*}$ by

$$
\begin{gathered}
\phi^{(1)}(x, y):=\left\{\begin{array}{ll}
\phi(x, y) & \text { in } \Omega_{1}, \\
0 & \text { in } \Omega \backslash \Omega_{1},
\end{array} \quad \phi^{(2)}(x, y):=\left\{\begin{array}{ll}
\phi(x, y) & \text { in } \Omega_{2}, \\
0 & \text { in } \Omega \backslash \Omega_{2},
\end{array},\right.\right. \\
\phi^{*}:=\phi^{(1)}+\alpha \phi^{(2)}(\alpha \in \mathbb{R}),
\end{gathered}
$$

where $\alpha$ is chosen such that $\left\langle\phi^{*}, \psi_{1}\right\rangle=0$ and $\psi_{1}$ is an eigenfunction of $\left(\mathrm{EPSE}_{\Omega}\right)$ corresponding to the first eigenvalue. Using the assumption of $\Omega_{1}$ and $\Omega_{2}$, we have

$$
\mu_{2}(\Omega) \geq \mathcal{H}_{\Omega}\left[\phi^{*}\right] /\left\|\phi^{*}\right\|_{L^{2}(\Omega)}^{2}=0 .
$$

We show by contradiction that $\mu_{2}(\Omega)>0$. Suppose that $\mu_{2}(\Omega)=0$. Then $\phi^{*}$ is an eigenfunction of $\left(\operatorname{EPSE}_{\Omega}\right)$. However, there is an interior point $p$ such that $\phi^{*}$ vanishes in a neighborhood of $p$. By the usual strong unique continuation at an interior point we see that $\phi^{*} \equiv 0$ in $\Omega$, which is a contradiction. Thus $\mu_{2}(\Omega)>0$.

We define $\partial_{\theta}^{\left(x_{1}, y_{1}\right)}$ by

$$
\partial_{\theta}^{\left(x_{1}, y_{1}\right)}:=-\left(y-y_{1}\right) \partial_{x}+\left(x-x_{1}\right) \partial_{y} .
$$


We denote $w_{\theta}$ by $\partial_{\theta}^{(0,0)} w$. Since $\partial_{\theta}^{\left(x_{1}, y_{1}\right)} \Delta=\Delta \partial_{\theta}^{\left(x_{1}, y_{1}\right)}$, we easily see that

$$
D_{u} \Delta\left(\partial_{\theta}^{\left(x_{1}, y_{1}\right)} w\right)+N^{\prime}(w)\left(\partial_{\theta}^{\left(x_{1}, y_{1}\right)} w\right)=0,
$$

provided that $w$ is a solution to $\left(\mathrm{SE}_{\Omega}\right)$.

Lemma 4.4. Let $\Omega\left(\subset \mathbb{R}^{2}\right)$ be a bounded convex domain with boundary of class $C^{2}$, and let $w \in C^{1}(\bar{\Omega})$ be a function such that $\partial_{\nu} w=0$ on $\partial \Omega$. Suppose that $\left(x_{0}, y_{0}\right) \in \partial \Omega$. Then

$$
\left(\partial_{\tau} w\right)\left(x_{0}, y_{0}\right)=0 \quad \text { if and only if } \quad\left(\partial_{\theta}^{\left(x_{1}, y_{1}\right)} w\right)\left(x_{0}, y_{0}\right)=0 \text { for all }\left(x_{1}, y_{1}\right) \in \operatorname{int}(\Omega) .
$$

In particular,

$$
\begin{aligned}
& \left(\partial_{\theta}^{\left(x_{1}, y_{1}\right)} w\right)\left(x_{0}, y_{0}\right)=0 \text { for some }\left(x_{1}, y_{1}\right) \in \operatorname{int}(\Omega) \quad \text { if and only if } \\
& \qquad\left(\partial_{\theta}^{\left(x_{1}, y_{1}\right)} w\right)\left(x_{0}, y_{0}\right)=0 \text { for all }\left(x_{1}, y_{1}\right) \in \operatorname{int}(\Omega) .
\end{aligned}
$$

Proof. The tangent line of $\partial \Omega$ at $\left(x_{0}, y_{0}\right)$ is not parallel to the vector $\left(x_{1}-x_{0}, y_{1}-y_{0}\right)$, because $\Omega$ is convex. Hence if $\left(\partial_{\theta}^{\left(x_{1}, y_{1}\right)} w\right)\left(x_{0}, y_{0}\right)=\left(\partial_{\nu} w\right)\left(x_{0}, y_{0}\right)=0$, then $w_{x}\left(x_{0}, y_{0}\right)=$ $w_{y}\left(x_{0}, y_{0}\right)=0$. Therefore $\left(\partial_{\tau} w\right)\left(x_{0}, y_{0}\right)=0$. Conversely, if $\left(\partial_{\tau} w\right)\left(x_{0}, y_{0}\right)=\left(\partial_{\nu} w\right)\left(x_{0}, y_{0}\right)$ $=0$, then $w_{x}\left(x_{0}, y_{0}\right)=w_{y}\left(x_{0}, y_{0}\right)=0$. Thus $\left(\partial_{\theta}^{\left(x_{1}, y_{1}\right)} w\right)\left(x_{0}, y_{0}\right)=-\left(y_{0}-y_{1}\right) w_{x}\left(x_{0}, y_{0}\right)$ $+\left(x_{0}-x_{1}\right) w_{y}\left(x_{0}, y_{0}\right)=0$ for all $\left(x_{1}, y_{1}\right) \in \operatorname{int}(\Omega)$.

We study the shape of the solution of $\left(\mathrm{SE}_{B}\right)$ when the Morse index of the solution is one under the condition that the solution is symmetric.

The next lemma is the key technical lemma of the paper.

Lemma 4.5. Let $w$ be a non-constant solution to $\left(\mathrm{SE}_{B}\right)$, and let $\mu_{2}(B)$ be the second eigenvalue of $\left(\mathrm{EPSE}_{B}\right)$. Suppose that $w$ is symmetric with respect to the line $L$ containing the center of $B$. If there is a point $\left(x_{0}, y_{0}\right) \in \operatorname{int}(B)$ such that $w_{x}\left(x_{0}, y_{0}\right)=w_{y}\left(x_{0}, y_{0}\right)=0$, then $\mu_{2}(B)>0$.

Proof. Let $w$ be a non-constant solution of $\left(\mathrm{SE}_{B}\right)$. After a rotation transformation, $w_{x}=w_{y}=0$ at the point corresponding to $\left(x_{0}, y_{0}\right)$. Therefore, we can assume, without loss generality, that $w$ is symmetric with respect to the $y$-axis. Let $\phi:=\partial_{\theta}^{(0,0)} w$. We divide the proof in two cases.

Case 1 $\left(x_{0} \neq 0\right)$ : We consider the case that $\left(x_{0}, y_{0}\right) \in \operatorname{int}(B) \backslash\{(x, y) ; x=0\}$. First, we assume that $\phi \not \equiv 0$ in $B$. Since $w_{x}\left(x_{0}, y_{0}\right)=w_{y}\left(x_{0}, y_{0}\right)=0, \phi\left(x_{0}, y_{0}\right)=0$. Proposition 2.4 says that there is a nodal curve on each side of $B \backslash\{(x, y) ; x=0\}$ and that each curve should divide each side of $B \backslash\{(x, y) ; x=0\}$ into at least two subdomains. Thus $\phi$ has at least four nodal domains. On the other hand, 0 is an eigenvalue of $\left(\mathrm{EPSE}_{B}\right)$, and $\phi$ is an associated eigenfunction. By the Courant nodal theorem we see that there is an integer $n_{0}(\geq 4)$ such that $\mu_{n_{0}}(B)=0$, where $\left\{\mu_{n}(B)\right\}_{n=1}^{\infty}$ is the eigenvalues of $\left(\mathrm{EPSE}_{B}\right)$. Therefore $\mu_{2}(B)>\mu_{n_{0}}(B)=0$.

Second, we assume that $\phi \equiv 0$ in $B$. Then an eigenfunction of $\left(\mathrm{EPSE}_{B}\right)$ corresponding to the first eigenvalue is radially symmetric, i.e., $\partial_{\theta}^{(0,0)} \psi_{1} \equiv 0$ in $B$. Since $w$ is radially symmetric, $\left\langle\psi_{1}, w_{x}\right\rangle=0$. Moreover $\left.w_{x}\right|_{\partial B}=0$, because $w_{x}(x, y)=\left(\partial_{r} w\right)(x, y) x / r$, 
where $r \partial_{r}:=x \partial_{x}+y \partial_{y}$ and $r:=\sqrt{x^{2}+y^{2}}$. On the other hand, we have that

$$
\mathcal{H}_{B}\left[w_{x}\right]=-D_{u} \int_{\partial B} w_{x}\left(\partial_{\nu} w_{x}\right) d \sigma=0,
$$

where we use (3.2) and the fact that $\left.w_{x}\right|_{\partial B}=0$. By a variational characterization of the second eigenvalue (3.1) we have that

$$
\mu_{2}(B) \geq \mathcal{H}_{B}\left[w_{x}\right] /\left\|w_{x}\right\|_{L^{2}(\Omega)}^{2}=0 .
$$

We will show by contradiction that $\mu_{2}(B)>0$. Suppose the contrary. We assume that $\mu_{2}(B)=0$. Then $w_{x}$ is an eigenfunction corresponding to 0 , and satisfies the Neumann boundary condition. Therefore $w_{x}=\partial_{\nu} w_{x}=0$ on $\partial B$. By a strong unique continuation on the boundary (Proposition 2.6) we see that $w_{x} \equiv 0$ in a neighborhood of a point on $\partial B$. Moreover, it follows from the strong unique continuation at an interior point that $w_{x} \equiv 0$ in $B$. Thus $w_{x}$ depends only on $y$. Since $w$ is radially symmetric, $w$ should be constant, which is a contradiction.

Case 2( $\left.x_{0}=0\right)$ : We consider the case that $\left(x_{0}, y_{0}\right) \in \operatorname{int}(B) \cap\{(x, y) ; x=0\}$. Let $\tilde{\phi}:=\partial_{\theta}^{\left(0, y_{0}\right)} w$. Then $\tilde{\phi}$ satisfies $D_{u} \Delta \tilde{\phi}+N^{\prime}(w) \tilde{\phi}=0$ in $B$, and the zero level set of $\tilde{\phi}$ is symmetric with respect to the $y$-axis. On the other hand, we have that

$$
\begin{aligned}
\tilde{\phi}(x, y) & :=-\left(y-y_{0}\right) w_{x}(x, y)+x w_{y}(x, y), \\
\tilde{\phi}_{x}(x, y) & =-\left(y-y_{0}\right) w_{x x}(x, y)+x w_{y y}(x, y), \\
\tilde{\phi}_{y}(x, y) & =-w_{x}(x, y)-\left(y-y_{0}\right) w_{x y}(x, y)+x w_{y y}(x, y) .
\end{aligned}
$$

Since $w_{x}\left(0, y_{0}\right)=w_{y}\left(0, y_{0}\right)=0$, we have that

$$
\tilde{\phi}\left(0, y_{0}\right)=\tilde{\phi}_{x}\left(0, y_{0}\right)=\tilde{\phi}_{y}\left(0, y_{0}\right)=0 .
$$

By Corollary 2.5 we see that either $\tilde{\phi} \equiv 0$ in $B$ or $\tilde{\phi}$ has at least four branches of $\{(x, y) ; \tilde{\phi}(x, y)=0\}$ at $\left(0, y_{0}\right)$. If $y_{0}=0$ and if $\tilde{\phi} \equiv 0$, then this case is already studied in Case 1. If $y_{0} \neq 0$ and if $\tilde{\phi} \equiv 0$, then we can easily show that $w$ is constant. We omit the details. Therefore we consider the case that $\tilde{\phi} \not \equiv 0$. Since the zero level set of $\tilde{\phi}$ is symmetric with respect to the $y$-axis, there is at least one branch on each side of $B \backslash\{(x, y) ; x=0\}$. First, we consider the case that each branch connects to $\bar{B} \cap\{(x, y) ; x=0\}$. Then there are two nodal domains $B_{1}, B_{2}$ such that $\partial B_{j} \cap(\partial B \backslash\{(x, y) ; x=0\})=\emptyset$ for $j=1,2$, and there is an interior point $p \in \operatorname{int}(B)$ such that $p \in \overline{B_{1}} \cup \overline{B_{2}}$. By Lemma 4.3 we see that $\mu_{2}(B)>0$.

Second, we consider the case that each branch does not connect to $\partial B \cap\{(x, y) ; x=0\}$. Then each branch should connect to $\partial B \backslash\{(x, y) ; x=0\}$. Let $p_{1}, p_{2} \in \partial B \backslash\{(x, y) ; x$ $=0\}$ be such intersection points. Then by Lemma 4.4 we see that $\phi\left(p_{1}\right)=\phi\left(p_{2}\right)=0$, because $\tilde{\phi}\left(p_{1}\right)=\tilde{\phi}\left(p_{2}\right)=0$. Moreover Proposition 2.7 says that there are nodal curves connecting to $p_{1}$ and $p_{2}$. Since the zero level set of $\phi$ is symmetric with respect to the $y$-axis, $\phi$ has at least four nodal domains. By the same argument as in the first part of Case 1 we see that $\mu_{2}(B)>0$.

Lemma 4.6. Let $w$ be a non-constant solution to $\left(\mathrm{SE}_{B}\right)$, and let $\mu_{2}(B)$ be the second eigenvalue of $\left(\operatorname{EPSE}_{B}\right)$. Suppose that $w$ is symmetric with respect to the line $L$ containing 
the center of $B$. Let $\partial_{\alpha} w$ denote the derivative in the direction parallel to $L$. If there exists a point $\left(x_{0}, y_{0}\right) \in \operatorname{int}(B)$ such that $\left(\partial_{\alpha} w\right)\left(x_{0}, y_{0}\right)=0$, then $\mu_{2}(B)>0$.

Proof. Because of the assumption of the lemma, we can assume, without loss of generality, that $w$ is symmetric with respect to the $y$-axis. Hence we see that $w_{x}=0$ on $B \cap\{(x, y) ; x=0\}$ and that

the zero level set of $w_{y}$ is symmetric with respect to the $y$-axis.

First, we show that if $w_{y}=0$ at a point on $\operatorname{int}(B) \cap\{(x, y) ; x=0\}$, then $\mu_{2}(B)>0$. Suppose that there is $\left(0, y_{0}\right) \in \operatorname{int}(B) \cap\{(x, y) ; x=0\}$ such that $w_{y}\left(0, y_{0}\right)=0$. Since $w_{x}\left(0, y_{0}\right)=w_{y}\left(0, y_{0}\right)=0$, we see by Lemma 4.5 that $\mu_{2}(B)>0$. From now on we assume that

$$
w_{y}>0 \text { on } \operatorname{int}(B) \cap\{(x, y) ; x=0\} .
$$

Second, we show that $w_{y}>0$ on $\partial B \backslash\{(x, y) ; x=0\}$. We see that $w_{x}=0$ at $\partial B \cap\{(x, y) ; x=0\}$. Thus $\partial_{\theta}^{(0,0)} w=0$ at $\partial B \cap\{(x, y) ; x=0\}$. Suppose that there is a point $p \in \partial B \backslash\{(x, y) ; x=0\}$ such that $\partial_{\theta}^{(0,0)} w=0$ at $p$. Then it follows from Proposition 2.7 that there is a nodal curve connecting to $p$. Therefore $\partial_{\theta}^{(0,0)} w$ has at least four nodal domains. By the Courant nodal theorem we see that there is an integer $n_{0}(\geq 4)$ such that $\mu_{n_{0}}(B)=0$. Thus $\mu_{2}(B)>\mu_{n_{0}}(B)=0$. Therefore $\partial_{\theta}^{(0,0)} w(x, y) \neq 0$ on $\partial B \backslash\{(x, y) ; x=0\}$. Suppose that there is a point $\left(x_{1}, y_{1}\right) \in \partial B \backslash\{(x, y) ; x=0\}$ such that $w_{y}\left(x_{1}, y_{1}\right)=0$. Since $\partial_{\nu} w=0$ on $\partial B, r \partial_{r} w\left(=x w_{x}+y w_{y}\right)=0$ on $\partial B$, $w_{x}\left(=-y / x w_{y}\right)=0$ at $\left(x_{1}, y_{1}\right)$. Hence $w_{\theta}\left(x_{1}, y_{1}\right)=0$, which is a contradiction. Therefore

$$
w_{y}>0 \text { on } \partial B \backslash\{(x, y) ; x=0\} .
$$

Third, we show by contradiction that $w_{y}>0$ in $\operatorname{int}(B) \backslash\{(x, y) ; x=0\}$. Suppose the contrary. We assume that there is a point $\left(x_{0}, y_{0}\right) \in \operatorname{int}(B) \backslash\{(x, y) ; x=0\}$ such that $w_{y}\left(x_{0}, y_{0}\right)=0$. Using (4.4), (4.5), (4.6) and Proposition 2.4, we see that there are two nodal domains, $B_{1}$ and $B_{2}$, and an interior point $p \in \operatorname{int}(B)$ such that $p \notin \overline{B_{1}} \cup \overline{B_{2}}$ and that $\left(\partial B_{j} \cap \partial B\right) \backslash\{(x, y) ; x=0\}=\emptyset$ for $j=1,2$. By Lemma 4.3 we see that $\mu_{2}(B)>0$.

The next theorem is our main result in the case that the domain is a ball.

Theorem 4.7 (Ball). Let $\Omega=B$, and let $(u, \xi)$ be a non-constant steady state to $\left(\mathrm{SS}_{B}\right)$. Suppose that $(\mathrm{N})$ and $\left(\mathrm{F}_{B}\right)$ hold. If $(u, \xi)$ is stable for some $\tau>0$, then $B$ has a diameter $P Q$ such that

(i ) $u$ is symmetric with respect to $P Q$,

(ii) $u$ is strictly monotone in the direction parallel to $P Q$, i.e., $\partial_{\alpha} u>0$ on $B \backslash\{P, Q\}$, where $\partial_{\alpha} u$ denotes the derivative in the direction,

(iii) $u_{\theta}>0$ on one side of $B \backslash \overline{P Q}, u_{\theta}<0$ on the other side, where $\overline{P Q}$ denotes the segment whose endpoints are $P$ and $Q$,

(iv) $u(Q)<u(x, y)<u(P)$ for $(x, y) \in B \backslash\{P, Q\}$.

Proof. Since $(u, \xi)$ is stable for some $\tau>0$, we see by Propositions 2.1 that $\chi_{2} \leq 0$, where $\chi_{2}$ is the second eigenvalue of $\left(\mathrm{LP}_{B}\right)$. Thus we see by Lemma 4.1 that there is a line $L$ containing the center of $B$ such that $u$ is symmetric with respect to $L$. Let 
$\phi:=\partial_{\theta}^{(0,0)} u$. By the same argument as in the second part of Case 1 in the proof of Lemma 4.5 we see that $\phi \not \equiv 0$ in $B$. Since $u$ is symmetric with respect to $L, L$ is a nodal line of $\phi$. We show by contradiction that there is no other nodal curve. Suppose that there is a point $p \in \bar{B} \backslash L$ such that $\phi=0$ at $p$. We can see by Propositions 2.4 and 2.7 that there are at least four nodal domains of $\phi$. By the Courant nodal theorem we see that there is an integer $n_{0}(\geq 4)$ such that $\chi_{n_{0}}=0$. Thus $\chi_{2}>\chi_{n_{0}}=0$, which contradicts $\chi_{2} \leq 0$. Thus $\phi$ has exactly two nodal domains.

Let $P, Q$ be intersection points of $\partial B$ and $L$. Using Lemma 4.1 and 4.6, we easily see that (i), (ii) and (iii) hold. Moreover, (iv) follows from Lemma 4.5

The next theorem is the main result of the case of an annulus.

Theorem 4.8 (Annulus). Let $\Omega=A\left(=B_{r_{1}} \backslash \overline{B_{r_{0}}}\right)$, and let $(u, \xi)$ be a steady state to $\left(\mathrm{SS}_{\Omega}\right)$. Suppose that $(\mathrm{N})$ and $\left(\mathrm{F}_{A}\right)$ holds. If $(u, \xi)$ is stable for some $\tau>0$, then there is a line, say $L$, containing the center of $B_{r_{1}}$ such that

(i ) $u$ is symmetric with respect to the line $L$,

(ii) $u_{\theta}>0$ on one side of $\bar{A} \backslash L, u_{\theta}<0$ on the other side,

(iii) $u$ attains its global maximum (minimum) on $\bar{A} \cap L$.

Proof. (i) follows from Remark 4.2. By the same argument as in the proof of Theorem 4.7 we see that (ii) holds. Using (ii), we easily see that the global maximum and the global minimum of $u$ are attained on $\bar{A} \cap L$. Therefore (iii) holds.

REMARK 4.9. It is known that if $\sup _{\rho \in \mathbb{R}}\left\{N^{\prime}(\rho) / \kappa_{2}(\Omega)\right\}<D_{u}$, then all the solutions of $\left(\mathrm{SE}_{\Omega}\right)$ are constant (see [CHS78, GM88]). Therefore Theorems 4.7 and 4.8 have meaning when

$$
\sup _{\left(\rho_{1}, \rho_{2}\right) \in \mathbb{R}^{2}}\left\{f_{u}\left(\rho_{1}, \rho_{2}\right) / \kappa_{4}(\Omega)\right\}<D_{u} \leq \sup _{\left(\rho_{1}, \rho_{2}\right) \in \mathbb{R}^{2}}\left\{f_{u}\left(\rho_{1}, \rho_{2}\right) / \kappa_{2}(\Omega)\right\}
$$

\section{Discussions.}

5.1. An extension to convex domains. We consider the case of two-dimensional bounded convex domain. As stated in the introduction, we can expect that the following holds: if $(u, \xi)$ is stable for some $\tau>0$, then the global maximum and minimum of $u$ are attained on the boundary of the domain. The following conjecture about the relation between the shape of the solution and the positivity of the second eigenvalue seems to be important in proving this:

ConjeCture 5.1 ([Y06]). Let $\Omega$ be a two-dimensional bounded convex domain with smooth boundary, and let $w$ be a non-constant solution of $\left(\operatorname{SE}_{\Omega}\right)$. Let $\mu_{2}(\Omega)$ denote the second eigenvalue of $\left(\operatorname{EPSE}_{\Omega}\right)$. If there is an interior point $\left(x_{0}, y_{0}\right) \in \operatorname{int}(\Omega)$ such that $\left(x_{0}, y_{0}\right)$ is a critical point of $w$, i.e., $w_{x}\left(x_{0}, y_{0}\right)=w_{y}\left(x_{0}, y_{0}\right)=0$, then $\mu_{2}(\Omega)>0$.

In the case that the domain is a two-dimensional ball (Lemma 4.5), we use the symmetry of the solutions in order to prove this conjecture.

This conjecture is a non-linear version of the "hot spots" conjecture of J. Rauch R74] which is the following: if the domain is convex, then an eigenfunction corresponding to the second eigenvalue of the Neumann Laplacian attains its global maximum on the boundary of the domain. There are counterexamples of the "hot spots" conjecture if the domain is a two-dimensional domain with one hole [B05] or two holes [BW99]. See 
[BB99, JN00] for partial positive answers of the conjecture. The "hot spot" conjecture follows from Conjecture 5.1 .

We can consider a more general problem. Let $\Omega$ be a convex domain. It is well-known that all the solutions of $\left(\mathrm{SE}_{\Omega}\right)$ are constant provided that $\mu_{1}(\Omega) \leq 0$ [CH78, Ma79]. If Conjecture 5.1 is correct, then all the solutions of $\left(\mathrm{SE}_{\Omega}\right)$ do not have a critical point in $\operatorname{int}(\Omega)$ provided that $\mu_{2}(\Omega) \leq 0$. For each $n \in\{1,2, \cdots\}$, when $\mu_{n}(\Omega) \leq 0$, what shape are the solutions of $\left(\mathrm{SE}_{\Omega}\right)$ ?

5.2. A relation between GM88 and our theorems. In GM88, Gurtin and Matano study the shape of the global and local minimizers of

$$
E[u]:=\int_{\Omega}\left\{\sigma|\nabla u(x)|^{2}+W(u(x))\right\} d x
$$

subject to the constraint

$$
\int_{\Omega} u(x) d x=m
$$

in various domains $\Omega$. Here $\sigma$ is a positive constant, $m$ is a constant and $W$ is the so-called double-well potential. A typical example of $W$ is given by $W^{\prime}(u)=u(1-u)(u-a)(0<$ $a<1)$. When the domain $\Omega$ is a two-dimensional ball, they obtain the same conclusion as Theorem 4.7 in the present paper under the assumption that a critical point $u$ of (5.1) subject to (5.2) is the global minimizer. (They also obtain results similar to Theorems 3.3 and 4.8 in the present paper when $\Omega$ is a rectangle or an annulus. See theorems in Sections 3 and 4 of GM88.)

Roughly speaking, the constraint (5.2) has the effect of removing the first eigenvalue in their problem [Ma05. Hence, if a critical point of (5.1) subject to (5.2) is a local minimizer, then the second eigenvalue is not positive. This is the same situation as ours. This is a reason why theorems in Sections 3 and 4 of [GM88, are similar to Theorems 3.3 . 4.7 and 4.8 of the present paper. However, from a technical point of view, the proofs of our results and theirs are different. In [GM88, the rearrangement technique is used when $\Omega$ is a two-dimensional ball. Therefore a critical point of (5.1) subject to (5.2) has to be assumed to be the global minimizer. On the other hand, we do not use this type of global assumption, which is difficult to check, and we use only a local property of the stability.

Acknowledgement. The author is grateful to Professor H. Matano for attracting his attention to the paper GM88 and Professor E. Yanagida for helpful comments. The main part of the paper was done when the author was at Hokkaido University. The author wants to express his gratitude to Professor Y. Nishiura at Hokkaido University for having given him a good research environment.

\section{REFERENCES}

[B05] K. Burdzy, The hot spots problem in planar domains with one hole, Duke Math. J., 129(2005), 481-502. MR 2169871

[BB99] R. Banuelos and K. Burdzy, On the "hot spots" conjecture of J. Rauch, J. Funct. Anal., 164(1999), 1-33. MR1694534 (2000m:35085)

[BW99] K. Burdzy and W. Werner, A counterexample to the "hot spots" conjecture, Ann. of Math. 149(1999), 309-317. MR.1680567 (2000b:35044) 
[C33] T. Carleman, Sur Les systèms linéaires aux derivées partielles du premier ordre á deux variables, C. R. Acad. Sci. Paris, 197(1933), 471-474.

[CH78] R. Casten and R. Holland, Instability results for reaction diffusion equations with Neumann boundary conditions, J. Diff. Eq., 27(1978), 266-273. MR480282 (80a:35064)

[CHS78] E. Conway, D. Hoff and J. Smoller, Large time behavior of solutions of systems of nonlinear reaction-diffusion equations, SAIM J. Appl. Math., 35(1978), 1-16. MR0486955 (58:6637)

[F61] R. FitzHugh, Impulses and physiological states in theoretical models of nerve membrane, Bilphys. J., 1(1961), 445-466.

[GM72] A. Gierer and H. Meinhardt, A theory of biological pattern formation, Kybernetik (Berlin), 12(1972), 30-39.

[GM88] M. E. Gurtin and H. Matano, On the structure of equilibrium phase transitions within the gradient theory of fluids, Quart. Appl. Math., 46(1988), 301-317. MR950604 (89j:49015)

[GW00] C. Gui, and J. Wei, On multiple mixed interior and boundary peak solutions for some singularly perturbed Neumann problems, Canad. J. Math., 52(2000), 522-538. MR.1758231 (2001b:35023)

[HMN99] T. Hoffmann-Ostenhof, P. Michor and N. Nadirashvili, Bounds on the multiplicity of eigenvalues for fixed membranes, Geom. Funct. Anal., 9(1999), 1169-1188. MR1736932(2001i:35066)

[HW53] P. Hartman and A. Wintner, On the local behavior of solutions of non-parabolic partial differential equations, Amer. J. Math., 75(1953), 449-476. MR0058082 (15:318b)

[JM94] S. Jimbo and Y. Morita, Stability of nonconstant steady-state solutions to a GinzburgLandau equation in higher space dimensions, Nonlinear Anal., 22(1994), 753-770. MR.1270168 (95i:35034)

[JN00] D. Jerison and N. Nadirashvili, The "hot spots" conjecture for domains with two axes of symmetry, J. Amer. Math. Soc., 13(2000), 741-772. MR.1775736 (2001f:35110)

[K05] M. Kuwamura, On the Turing patterns in one-dimensional gradient/skew-gradient dissipative systems, SIAM J. Appl. Math., 65(2004/05), 618-643. MR2123072 (2006b:35190)

[KY03] M. Kuwamura and E. Yanagida, The Eckhaus and zigzag instability criteria in gradient/skewgradient dissipative systems, Phys. D, 175(2003), 185-195. MR.1963859 (2004c:35163)

[L96] O. Lopes, Radial and nonradial minimizers for some radially symmetric functions, Elec. J. Diff. Eq., 1996, 1-14. MR.1375123 (96m:90078)

[L01] C. S. Lin, Locating the peaks of solutions via the maximum principle: I. The Neumann problem, Comm. Pure Appl. Math., 54(2001), 1065-1095. MR.1835382(2002d:35052)

[LNT88] C. S. Lin, W. M. Ni and I. Takagi, Large amplitude stationary solutions to a chemotaxis system, J. Diff. Eq., 72(1988), 1-27. MR929196 (89e:35075)

[LT01] C. S. Lin and I. Takagi, Method of rotating planes applied to a singularly perturbed Neumann problem, Calc. Var. Partial Differential Equations, 13(2001), 519-536. MR.1867940 (2002k:35116)

[Ma79] H. Matano, Asymptotic behavior and stability of solutions of semilinear diffusion equations, Publ. Res. Inst. Math. Sci., 15(1979), 401-454. MR555661 (80m:35046)

[Ma05] H. Matano, private communication, (2005).

[Mi05] Y. Miyamoto, Stability of a boundary spike layer for the Gierer-Meinhardt system, Euro. J. Appl. Math., 16(2005), 467-491. MR2195264(2006j:35135)

[Mi06] Y. Miyamoto, An instability criterion for activator inhibitor systems in a two-dimensional ball, to appear in J. Diff. Eq.

[MM02] A. Malchiodi and M. Montenegro, Boundary concentration phenomena for a singularly perturbed elliptic problem, Comm. Pure Appl. Math., 55(2002), 1507-1568. MR.1923818 (2003g:35005)

[N82] Y. Nishiura, Global structure of bifurcating solutions of some reaction-diffusion systems, SIAM J. Math. Anal., 13(1982), 555-593. MR661590 (83h:58029)

[N94] Y. Nishiura, Coexistence of infinitely many stable solutions to reaction diffusion systems in the singular limit, Dynamics Reported, 3(1994), 25-103.

[NAY62] J. Nagumo, S. Arimoto and S. Yoshizawa, An active pulse transmission line simulating nerve axon, Proc. Inst. Radio. Engineers, 50(1962), 2061-2070.

[NPY01] W. M. Ni, P. Poláčik and E. Yanagida, Monotonicity of stable solutions in shadow systems, Trans. Amer. Math. Soc., 353(2001), 5057-5069. MR1852094 (2002e:35132)

[NT91] W. M. Ni and I. Takagi, On the shape of least energy solution to a semilinear Neumann problem, Comm. Pure Appl. Math., 41(1991), 819-851. MR.1115095(92i:35052) 
[NT93] W. M. Ni and I. Takagi, Locating the peaks of least energy solutions to a semilinear Neumann problem, Duke. Math. J., 70(1993), 247-281. MR.1219814 (94h:35072)

[NTY01] W. M. Ni, I. Takagi and E. Yanagida, Stability of least energy patterns of the shadow system for an activator-inhibitor model, Japan J. Indust. Appl. Math., 18(2001), 259-272. MR.1842911 (2002g:35120)

[R74] J. Rauch, Five problems: an introduction to the qualitative theory of partial differential equations, Partial Differential Equations and Related Topics (Jerome A. Goldstein, ed.), SpringerVerlag, Berlin, 1974, 355-369, Lecture Notes in Mathematics 446. MR0509045 (58:22963)

[Y02a] E. Yanagida, Mini-maximizers for reaction-diffusion systems with skew-gradient structure, J. Diff. Eq., 179(2002), 311-335. MR.1883746 (2002j:35184)

[Y02b] E. Yanagida, Standing pulse solutions in reaction-diffusion systems with skew-gradient structure, J. Dynam. Diff. Eq., 14(2002), 189-205. MR1878648(2002k:35176)

[Y02c] E. Yanagida, Stability analysis for shadow systems with gradient/skew-gradient structure, International Conference on Reaction-Diffusion Systems: Theory and Applications (Kyoto, 2001). Sūrikaisekikenkyūsho Kōkyūroku, 1249(2002), 133-142. MR 1924188

[Y06] E .Yanagida, private communication, (2006). 